\title{
BMJ Cerebral blood flow velocity changes Open during upright positioning in bed after acute stroke: an observational study
}

\author{
Marcel J Aries, ${ }^{1}$ Jan Willem Elting, ${ }^{1}$ Roy Stewart, ${ }^{2}$ Jacques De Keyser, ${ }^{1,3}$ \\ Berry Kremer, ${ }^{1}$ Patrick Vroomen ${ }^{1}$
}

To cite: Aries MJ, Elting JW, Stewart R, et al. Cerebral blood flow velocity changes during upright positioning in bed after acute stroke: an observational study. BMJ Open 2013;3:e002960. doi:10.1136/bmjopen-2013002960

- Prepublication history and additional material for this paper is available online. To view these files please visit the journal online (http://dx.doi.org/10.1136/ bmjopen-2013-002960).

Received 27 March 2013 Revised 17 July 2013 Accepted 19 July 2013

${ }^{1}$ Department of Neurology, University of Groningen, University Medical Center Groningen, Groningen, The Netherlands

${ }^{2}$ Health Sciences, Community and Occupational Medicine, University of Groningen, University Medical Center Groningen, Groningen, The Netherlands ${ }^{3}$ Center for Neurosciences Vrije Universiteit Brussel (VUB), Brussels, Belgium

Correspondence to Dr Marcel Aries; m.j.h.aries@umcg.nl

\section{ABSTRACT}

Objectives: National guidelines recommend mobilisation in bed as early as possible after acute stroke. Little is known about the influence of upright positioning on real-time cerebral flow variables in patients with stroke. We aimed to assess whether cerebral blood flow velocity (CBFV) changes significantly after upright positioning in bed in the acute stroke phase.

Design: Observational study.

Participants: 47 patients with acute ischaemic stroke measured in the subacute phase after symptom onset and 20 healthy controls.

Primary and secondary outcome measures: We recorded postural changes in bilateral transcranial Doppler (primary outcome) and simultaneously recorded nearinfrared spectroscopy, end-tidal $\mathrm{CO}_{2}$, non-invasive blood pressure data and changes in neurological status (secondary outcomes).

Methods: Postures included the supine, half sitting $\left(45^{\circ}\right)$, sitting $\left(70^{\circ}\right)$ and Trendelenburg $\left(-15^{\circ}\right)$ positions. Using multilevel analyses, we compared postural changes between hemispheres, outcome groups (using modified Rankin Scale) as well as between patients and healthy controls.

Results: The mean patient age was $62 \pm 15$ years and median National Institute of Health Stroke Scale score on admission was 7 (IQR 5-14). Mean proportional CBFV changes on sitting were not significantly different between healthy controls and affected hemispheres in patients with stroke. No significant differences were found between affected and unaffected stroke hemispheres and between patients with unfavourable and favourable outcomes. During upright positioning, no neurological worsening or improvement was observed in any of the patients.

Conclusions: No indications were found that upright positioning in bed in mild to moderately affected patients with stroke compromises flow and (frontal)oxygenation significantly during the subacute phase of stroke. Supine or Trendelenburg positioning does not seem to augment real-time flow variables.

\section{INTRODUCTION}

Brain ischaemia is a potentially reversible process that is dependent on restoration of

\section{ARTICLE SUMMARY}

Article focus

- Stroke units save more patients with stroke from death or dependency than any other intervention in stroke.

- Early upright positioning is an intervention in the stroke unit that is recommended in several national guidelines on the basis of presumed benefits, though evidence is lacking.

- This study aimed to assess whether cerebral blood flow velocity (CBFV) —as a surrogate marker for cerebral perfusion-changes significantly after upright positioning in bed in the subacute stroke phase.

\section{Key messages}

- Following acute ischaemic stroke in the middle cerebral artery territory, early upright positioning in bed resulted in minor average changes in CBFV and cerebral oxygenation only and therefore appears to be safe during the subacute phase on the stroke unit.

- Our work is the largest observational study about real-time postural haemodynamic changes so far and is important in further unravelling the benefits of stroke unit care and early mobilisation in particular.

Strengths and limitations of this study

- The study is an example of how a small-scale study of an important surrogate endpoint can help define stroke unit management when evidence from large-scale clinical trials is not yet available. We feel a wider application of such bedside techniques in similar studies in the stroke unit is also an important means to increase the gain of subsequent larger clinical trials.

- Replication of this study should be considered when studying the (hyper)acute stroke phase, longer sitting periods, active standing and using more sophisticated and direct methods that measure cerebral perfusion regionally and estimate cerebral oxygen metabolism.

cerebral blood flow (CBF) within a time window of cellular viability that varies according to the severity and duration of flow 
cessation. ${ }^{1}$ Any activity that alters CBF during the acute phase of ischaemic stroke may, at least theoretically, directly contribute to extension of the penumbra to the ischaemic core and compromise clinical outcome. National guidelines recommend mobilisation in and out of bed as early as possible after stroke. ${ }^{2-4}$ Such early upright positioning prevents choking, cardiopulmonary deconditioning and may improve early plasticity of the brain. ${ }^{5} 6$ However, there is insufficient evidence in support of early upright positioning after acute stroke. ${ }^{7}$ Also, there is fear that mobilisation in the setting of a (potentially)disturbed cerebral autoregulation leads to gravity induced diminished CBF to the penumbra and, potentially, extension of the infarct core. ${ }^{8}$ As a result, early upright positioning is not universally accepted by stroke physicians.

Little is known in patients with acute stroke about the influence of different body positions on blood pressure (BP) or on real-time cerebral flow variables. Transcranial Doppler (TCD) may be used to assess CBF velocity $(\mathrm{CBFV})$ at the stroke unit bedside. Nursing patients 'heads down' has been advocated, based on a $20 \%$ augmentation of $\mathrm{CBFV}$ when comparing supine with $30^{\circ}$ head elevation in 20 patients severely affected with stroke. ${ }^{9}$ If such relatively minor changes in head position have such an impact, what would the consequences of more pronounced changes in position be in a representative sample of stroke unit patients?

In this study, we sought to assess the impact of the various head positions on CBFV comparing (1) hemispheres affected by stroke and unaffected hemispheres and (2) affected hemispheres and healthy controls. Any effect of upright positioning on neurological status and outcome was explored.

\section{METHODS}

\section{Study design and patients}

This observational study was conducted in the stroke unit of a teaching hospital between August 2008 and May 2011. Patients aged over 18 years with a first ever ischaemic stroke in the middle cerebral artery (MCA) territory were considered eligible. The measurements were taken on the stroke unit and mainly because of practical reasons (installation on stroke unit, informed consent approval and no interference with urgent clinical studies or consultations) performed in the subacute phase. Our protocol dedicated that the measurements had to be completed between 6 and $16 \mathrm{~h}$ after stroke symptom onset, mimicking the period of early in bed mobilisation initiation during daytime. Patients had to be conscious, cooperative, haemodynamically stable without fever and display flow in both M1 segments of the MCA. Moreover, patients deemed to be too severely affected by the stroke or too severely ill to be positioned upright (clinical/nursing judgement) were not included in this study. Brain CT, ECG, bilateral duplex carotid ultrasound and laboratory investigations were performed. Thrombolytic therapy was administered according to (inter)national guidelines. Use of any medication, especially antiarrhythmics and antihypertensive medication, was registered. The National Institutes of Health Stroke Scale score (NIHSS) was used to determine neurological deficit. The stroke subtype was classified using the Oxfordshire Community Stroke Project classification, ${ }^{10}$ and stroke aetiology according to Trial of Org 10172 in Acute Stroke Treatment (TOAST) definitions. ${ }^{11}$ The modified Rankin Scale (mRS) score at 3 months was determined by telephone interview by a certified assessor blinded to study data. Twenty healthy controls were recruited at random from (retired) work associates and their medical histories were reviewed. All experiments complied with the tenets of the Declaration of Helsinki and the study protocol was reviewed and approved by our institutional review board. Study participants provided written informed consent. Next of kin gave consent when participants were unable to provide it.

\section{Sample size calculation}

In the literature, SD of the intraindividual supine to sitting MCA CBFV difference is estimated to be around $\pm 5 \%$ for elderly hospitalised patients ${ }^{12}$ and $\pm 20 \%$ for patients with severe stroke going from the $30^{\circ}$ to $0^{\circ}$ position. ${ }^{9}$ To detect a difference in mean CBFV of more than $6 \mathrm{~cm} / \mathrm{s}(10-20 \%)$ between remaining supine and sitting upright in bed with a probability (power) of $90 \%$ when testing at a two-sided significance level of $5 \%$, a sample size of 45 participants was required.

\section{Procedures}

BP was measured with a finger plethysmograph (Finometer-Pro, Finapress Medical Systems, Amsterdam, the Netherlands). The cuff was applied to the middle finger of the non-dominant or paretic hand placed at the heart level with the arm across the chest and supported by a sling. TCD was performed by the same investigator (MA) over the temporal bone window on both sides. MCA wave forms were identified at a depth range of 40-60 $\mathrm{mm}$ and a stable forward waveform with good intensity was selected for monitoring (Nicolet Pioneer TC8080, Carefusion Corporation, San Diego, California, USA). Patients with permanent MCA occlusion on TCD were excluded. TCD probes $(2 \mathrm{MHz})$ were mounted by a head frame to ensure a constant angle of insonation throughout the positioning procedure. Bifrontal nearinfrared spectroscopy (NIRS) recordings were added from 2010 onwards for regional cerebral oxygenation $\left(\mathrm{rSO}_{2}, \%\right)$ calculation. The INVOS 5100C NIRS device (Somanetics Corp, Detroit, Michigan, USA) has a sampling frequency of $0.2 \mathrm{~Hz}$. Adhesive optodes were placed on each side of the forehead. End-tidal $\mathrm{CO}_{2}\left(\mathrm{EtCO}_{2}\right.$, kPA) was measured by a mask using an infrared $\mathrm{CO}_{2}$ analyser (Capnomac Ultima, GE Healthcare, Chalfont St Giles, UK) as an approximation of partial arterial $\mathrm{CO}_{2}$ pressure. 


\section{Signals}

The signals of the $\mathrm{BP}$ and $\mathrm{EtCO}_{2}$ waveforms, spectral envelope of MCA CBFV, and $\mathrm{rSO}_{2}$ as well as the position marker signals were sampled at $250 \mathrm{~Hz}$ and synchronously captured using an A/D converter. Signals were recorded with LabVIEW software (LabVIEW V.7.1, National Instruments, Austin, Texas, USA) for online inspection and were stored for postprocessing. The MCA $\mathrm{CBFV}_{\text {mean }}(\mathrm{cm} / \mathrm{s})$ was calculated by using beat-to-beat mean values that were obtained by triggering off the ascending slope of the BP curve. Mean arterial pressure (MAP) at the MCA level $\left(\mathrm{MAP}_{\mathrm{mca}}, \mathrm{mm} \mathrm{Hg}\right.$ ) was automatically calculated from MAP measured at the heart level and the vertical finger-to-TCD probe distance. In the upright and Trendelenburg positions, great care was taken to secure the hand with the finger plethysmograph cuff at the heart level (approaching the right atrium level). The beginning and ending time points of the postural transitions were marked, but these data periods $( \pm 30 \mathrm{~s})$ were not used for analysis.

\section{Predefined positioning protocol}

After admission to the stroke unit, patients were instrumented. Participants were requested to abstain from moving and talking in order to minimise artefacts and activity-related CBFV changes. Great care was taken to keep the head in the neutral midline position (without shifting of the headband) and the BP cuff at the heart level during positioning. A test run was performed. After 5 min of supine rest (baseline (eg, first) supine), the participants were passively tilted by changing the head of the bed end angles to the $45^{\circ}$ (half sitting), $70^{\circ}$ (sitting) and $-15^{\circ}$ (Trendelenburg) positions for 3-5 min each. Patients were brought in the sitting position twice. Periods of tilts were interspaced with $3-5 \mathrm{~min}$ of supine rest to re-establish control values (supine position). Before and after establishing each position, the motor score of NIHSS (affected arm and leg) was evaluated. Control participants were recorded in a quiet, temperature-controlled research room using the same predefined positioning protocol.

\section{Statistical analysis}

We calculated a mean value for every position period. To compare responses in each position, percentage changes in the variables $\left(\mathrm{MCA} \mathrm{CBFV}_{\text {mean }}, \mathrm{rSO}_{2}, \mathrm{MAP}_{\text {mca }}\right.$, heart rate $(\mathrm{HR})$ and $\mathrm{EtCO}_{2}$ ) were calculated as the differences between the positions after normalisation to the mean baseline (eg, first) supine value. Normalisation was carried out to be able to compare physiological responses between patients, healthy controls and hemispheres, where absolute individual values might differ from baseline. We averaged these percentage changes to obtain a mean percentage change for each of the four different positions: supine, half sitting, sitting and Trendelenburg. For control participants, the mean of the $\mathrm{MCA} \mathrm{CBFV}_{\text {mean }}$ and $\mathrm{rSO}_{2}$ signals was taken. To account for and to take advantage of the correlation between repeated measures in the same individual, a multilevel model was used to estimate the effects of positioning and affected hemisphere on changes in the main variables $\left(\mathrm{MCA} \mathrm{CBFV}_{\text {mean }}\right.$ and $\left.\mathrm{rSO}_{2}\right) .{ }^{13}$ The relation between $\mathrm{MCA} \mathrm{CBFV}_{\text {mean }}$ and outcome (dichotomised as mRS 0-2 for favourable outcome) was analysed in a similar way. Statistical analysis was performed with SAS (V.9.2). Significant carotid stenosis, stroke subtype or aetiology (TOAST criteria), NIHSS, age and sex were used as potential confounding covariates in stroke group comparisons. ${ }^{14}$ Age, sex and history of hypertension, cardiac disease and diabetes were used as covariates for healthy control and stroke group comparisons. Bonferroni adjustments for multiple comparisons were used for all results presented.

\section{RESULTS}

In eight patients with subacute stroke, TCD or BP investigation was unsuccessful. Eventually, we enrolled 47 patients (table 1). The mean patient age was 62 \pm 15 years and median baseline NIHSS was 7 points (IQR 5-14 points). At 3 months, 29 patients $(62 \%)$ had a favourable outcome. NIRS recordings were performed in 32 patients $(68 \%)$ and 16 controls $(80 \%)$.

We compared the cerebral haemodynamic postural $\left(\mathrm{MCA} \mathrm{CBFV}_{\text {mean }}\right.$ and $\mathrm{rSO}_{2}$ ) changes between positions, patients with stroke and healthy controls, and between affected and unaffected hemispheres. A complete overview of the comparisons is presented in online supplementary tables 1-3 (http://bmjopen.bmj.com). Figure 1 shows that cerebral haemodynamic changes were most pronounced when moving from the supine to the sitting position $\left(70^{\circ}\right)$ for patients (affected hemisphere) and healthy controls (figure 1 ). The results presented below therefore focus on changes from the supine to the sitting position. The accompanying systemic haemodynamic ( $\mathrm{MAP}_{\text {mca }}$ and $\mathrm{HR}$ ) and respiratory responses $\left(\mathrm{EtCO}_{2}\right)$ are shown in figure 2.

In the sitting position for the affected and unaffected hemispheres, $\mathrm{MCA} \mathrm{CBFV}_{\text {mean }}$ decreased in 28 patients $(60 \%)$ and $\mathrm{rSO}_{2}$ decreased in 18 patients $(55 \%)$. None of the univariate results presented below were different after adjustment for predefined covariates, except for the outcome analysis.

\section{Positional CBFV changes on sitting in patients with stroke} The mean MCA $\mathrm{CBFV}_{\text {mean }}$ decreased significantly with movement to the sitting position, (mean difference $-4 \%$, $95 \%$ CI $-6 \%$ to $-3 \%$; $p=0.005)$ for the affected hemisphere. For the unaffected hemisphere, this effect was not significant (mean difference $-2 \%, 95 \%$ CI $-5 \%$ to $0 \%$; $\mathrm{p}=1.0$ ).

Positional CBFV changes on sitting comparing patients with stroke and healthy controls

The mean $\mathrm{MCA} \mathrm{CBFV}_{\text {mean }}$ changes on sitting were comparable between healthy controls and affected hemispheres of 
Table 1 Patients' demographics and clinical characteristics

\begin{tabular}{|c|c|c|}
\hline Variables & Patients with stroke $(n=47)$ & Healthy controls $(n=20)$ \\
\hline Mean age (SD), years & $62(15)$ & $52(20)$ \\
\hline Male & $32(70)$ & $11(55)$ \\
\hline Affected right hemisphere & $24(52)$ & - \\
\hline Median NIHSS score on admission (IQR) & $7(5-14)$ & - \\
\hline History of hypertension & $21(46)$ & $7(35)$ \\
\hline History of cardiac disease & $17(37)$ & $0(0)$ \\
\hline Significant carotid stenosis & $8(17)$ & Unknown \\
\hline \multicolumn{3}{|l|}{ Type infarct } \\
\hline Atherosclerotic & $15(33)$ & \\
\hline Cardiac & $10(22)$ & \\
\hline Small vessel & $14(30)$ & \\
\hline Dissection & $2(4)$ & \\
\hline Unknown & $5(11)$ & \\
\hline \multicolumn{3}{|l|}{ Oxford classification } \\
\hline Total anterior circulation infarction & $9(20)$ & \\
\hline Partial anterior circulation infarction & $19(41)$ & \\
\hline Lacunar circulation infarction & $18(40)$ & \\
\hline Posterior circulation infarction & $0(0)$ & \\
\hline History of diabetes mellitus & $8(17)$ & $0(0)$ \\
\hline Antihypertensive medication on admission & $25(54)$ & $6(30)$ \\
\hline Intravenous thrombolysis & $20(44)$ & - \\
\hline Mean arterial pressure (SD), $\mathrm{mm} \mathrm{Hg}$ & $84(21)$ & $78(13)$ \\
\hline Mean heart rate (SD), bpm & $77(18)$ & $64(7)$ \\
\hline
\end{tabular}

patients with stroke (mean difference $-4 \%$ for the affected hemisphere $(95 \%$ CI $-11 \%$ to $3 \% ; \mathrm{p}=1.0)$ as well as unaffected hemispheres (mean difference $-5 \%, 95 \%$ CI $-10 \%$ to $1 \%$; $\mathrm{p}=0.18)$ ).
Positional CBFV changes on sitting comparing hemispheres in patients with stroke

The mean MCA $\mathrm{CBFV}_{\text {mean }}$ was not different between the affected and the unaffected hemispheres in the
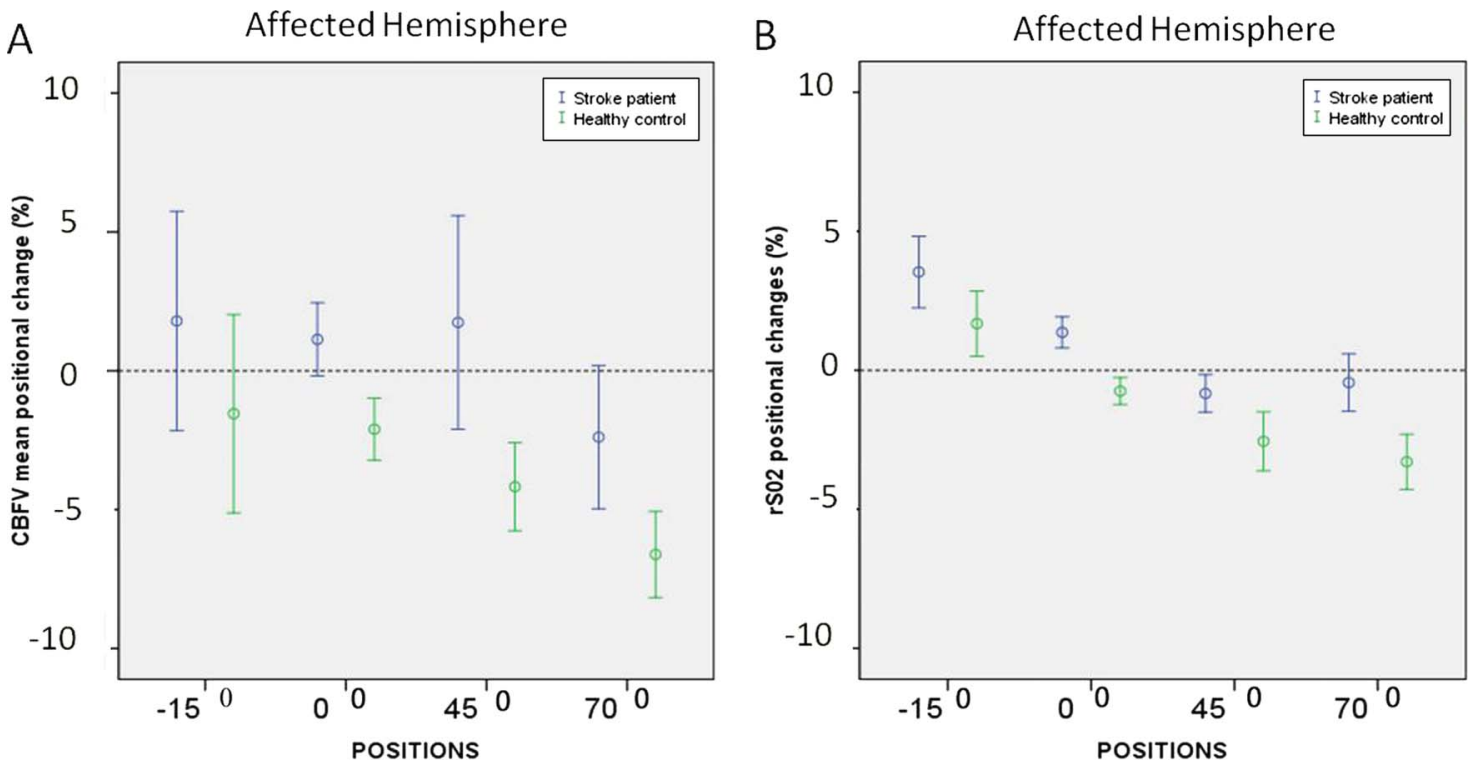

Figure 1 Cerebral haemodynamic changes in different body positions in patients with subacute stroke and healthy controls. Image showing changes of $\mathrm{MCA} \mathrm{CBFV}$ mean $(\mathrm{A})$ and $\mathrm{rSO}_{2}(\mathrm{~B})$ in different positions following subacute stroke in patients (affected hemisphere) and healthy controls. All data were normalised to the baseline (eg, first) supine position and displayed in percentages (\%). Thirty-two patients had near-infrared spectroscopy recordings. CBFV, cerebral blood flow velocity; MCA, middle cerebral artery; $\mathrm{rSO}_{2}$, regional cerebral oxygen saturation. 


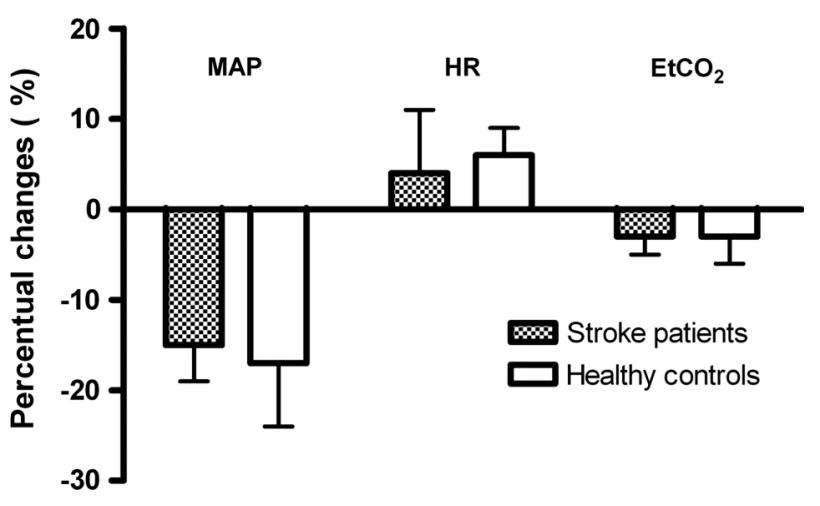

Figure 2 Systemic haemodynamic changes with going to the upright position in patients with subacute stroke and healthy controls. Image showing the changes of mean arterial pressure at the middle cerebral artery level $\left(\mathrm{MAP}_{\mathrm{mca}}\right)$, heart rate $(\mathrm{HR})$ and end-tidal $\mathrm{CO}_{2}\left(\mathrm{EtCO}_{2}\right)$ with going to the upright position $\left(70^{\circ}\right)$ in patients with subacute stroke and healthy controls. All data were normalised to the baseline (eg, first) supine position and displayed in percentages (\%).

sitting position (mean difference $-2 \%, 95 \%$ CI $-5 \%$ to $2 \% ; \mathrm{p}=1.0)$.

\section{Positional changes of $\mathrm{rSO}_{2}, \mathrm{MAP}_{\mathrm{mca}}, \mathrm{HR}$ and $\mathrm{EtCO}_{2}$ on sitting}

The mean $\mathrm{rSO}_{2}$ values decreased significantly with movement to the sitting position for the affected hemisphere (mean difference $-2 \%, 95 \%$ CI $-3 \%$ to $-1 \%$; $\mathrm{p}=0.006$ and unaffected hemisphere (mean difference $-2 \%, 95 \%$ CI $-3 \%$ to $-1 \% ; \mathrm{p}=0.009)$ ). In agreement with the $\mathrm{MCA} \mathrm{CBFV}_{\text {mean }}$ results, the mean $\mathrm{rSO}_{2}$ postural responses were not significantly different between both hemispheres and between patients and healthy controls. The $\mathrm{CBFV}_{\text {mean }}$ and $\mathrm{rSO}_{2}$ changes were positively correlated for the affected hemisphere $(\mathrm{r}=0.33, \mathrm{p}=0.01)$.

In patients with stroke, $\mathrm{MAP}_{\mathrm{mca}}$ decreased on average by $15 \%$ in the sitting position (95\% CI $-19 \%$ to $-10 \%$; $\mathrm{p}<0.001)$. This was similar to controls $(17 \%, 95 \%$ CI $-24 \%$ to $-11 \%$; $\mathrm{p}<0.001$; figure 2$)$. $\mathrm{MAP}_{\mathrm{mca}}$ increased slightly during the course of the experiment for patients (mean 5\%, 95\% CI 2\% to $7 \%$ ) and healthy controls (mean $2 \%, 95 \%$ CI $-1 \%$ to $6 \%$ ). The postural responses were accompanied by only minor $\mathrm{HR}$ changes. $\mathrm{EtCO}_{2}$ decreased on average by $3 \%(95 \%$ CI $-5 \%$ to $-1 \%$; $\mathrm{p}<0.001$ ) for patients in the sitting position.

\section{Positional changes, neurological status and relation with outcome in patients with stroke}

We observed no neurological worsening or improvement during the postural changes in any of the patients. There were no significant differences between the mean $\mathrm{MCA} \mathrm{CBFV}_{\text {mean }}$ changes on upright and Trendelenburg positioning in patients with unfavourable compared to favourable outcome after correction for confounding covariates (mean difference on sitting, for the affected hemisphere: $-5 \%, 95 \%$ CI $-14 \%$ to $5 \%$; $\mathrm{p}=1.0)$.

\section{DISCUSSION}

Our study showed that following an ischaemic stroke in the MCA territory, early upright positioning in bed resulted in minor average changes in CBFV only in the subacute phase. The postural responses were not significantly different between the affected and unaffected hemispheres, and between patients and healthy controls. Passive upright positioning was not accompanied by worsening of the (motor) stroke severity score. No improvement was noticed in the supine and Trendelenburg positions. The relative postural haemodynamic and $\mathrm{EtCO}_{2}$ changes were largely comparable between patients and controls. This study found no indications that upright positioning compromises flow or (frontal) oxygenation significantly in the affected MCA territory in mild to moderately affected patients with acute stroke. No association was found between flow changes and functional outcome. Our results suggest effective cerebral and/or systemic haemodynamic adjustments to overcome gravitational force during postural changes in the subacute phase of mild to moderate stroke. The potential of the Trendelenburg position to augment CBF seems to be limited and may not outweigh concurrent cardiopulmonary complications.

Although TCD measurements cannot be used to calculate $\mathrm{CBF}$, the observed relative $\mathrm{CBFV}$ changes can be considered proportionate to $\mathrm{CBF}$ changes as long as the angle of insonation and the MCA diameter remain constant during the brief test interval. The MCA diameter is thought to remain constant over a $\pm 30 \mathrm{~mm} \mathrm{Hg}$ range of $\mathrm{BP}$ and $\pm 2 \mathrm{kPA}$ of $\mathrm{CO}_{2} \cdot{ }^{15}$ This requirement was fulfilled in our study. TCD is prone to several errors because the measurements are operator dependent and patient dependent. Even small probe position changes may alter the CBFV readings. However, frontal cerebral oxygenation $\left(\mathrm{rSO}_{2}\right)$ followed CBFV changes in size and direction in the affected hemisphere, which further strengthens our findings. Assuming a constant arterial $\mathrm{O}_{2}$ content and cerebral metabolic rate during the measurement, $\mathrm{rSO}_{2}$ is predominantly a function of local CBF. ${ }^{16}$ Furthermore, TCD monitoring is the only non-invasive method to monitor CBF changes continuously at the bedside. It is generally accepted that CBF changes are closely reflected by TCD readings. ${ }^{18}$

Patients in our study predominantly had mild to moderate strokes. Patients with MCA occlusion were excluded. It was left to clinical and nursing judgement whether patients were deemed too severely affected by the stroke or too severely ill to be positioned upright for this study. Although NIHSS was not found to be a confounder for postural CBFV changes, too few patients with severe stroke (NIHSS >16) were included to be able to generalise our findings to patients with severe strokes. Furthermore, fully ambulatory patients may have been less likely to be included. The practical implication of the latter drawback in generalisation is probably limited. When mild to moderate strokes are unlikely to be affected by upright positioning, very mild strokes are even less likely to be affected. 
Three studies have investigated the postural effects on CBFV in acute stroke. ${ }^{9} 1920$ Wojner et al found on average a $20 \% \mathrm{CBFV}$ increase in the supine position in 20 patients with an acute MCA occlusion. However, a wide range of individual flow improvements was present $(5-96 \%$ from baseline), with no data from the unaffected hemisphere being available. ${ }^{9}$ A small study with eight participants found important postural flow changes in four severely affected patients with incomplete MCA recanalisation on $24 \mathrm{~h}$ follow-up imaging (affected hemisphere) ${ }^{19} \mathrm{~A}$ more than $15 \%$ higher MCA flow was found in the supine position in 18 patients with large stroke with intracranial pressure (ICP) monitoring in the intensive care unit. This effect was only present in the affected hemisphere. ${ }^{20}$ Different results were obtained in a single-photon emission CT (SPECT) stroke study. Seven of eight patients with subacute stroke had a clear visual improvement in SPECT perfusion to the affected frontal, temporal or parietal lobes in the semirecumbent $\left(30-45^{\circ}\right)$, compared to the supine position. No differences were found in BP in both positions. Unfortunately, no demographics, clinical effect or respiratory changes of the more upright position were reported. $^{21}$

Our study did not show relevant postural changes despite the larger position changes $\left(70^{\circ}\right.$ instead of $\left.30^{\circ}\right)$. This is probably largely due to our predominant focus on mild to moderately affected patients with stroke. Also, in our study, changes in all variables were related to the individual baseline supine level (by normalisation), reducing the effects of large variations of measured parameters and difficulties with standardisation of the used techniques (TCD, NIRS and BP). In none of the four studies were flow data recorded simultaneously with NIRS and $\mathrm{EtCO}_{2}$. In the study of Hargroves et al, cerebral oxygenation as measured by NIRS in the first seven days after stroke was lowest in the upright position and highest in the supine position (difference estimated around 2-6\%). These changes were also present in the unaffected hemisphere, although on visual inspection they were less marked. ${ }^{22}$ Furthermore, there might be an effect of anaesthesia, vasopressors, hypothermia and decompressive craniectomy on cerebral vasoregulation used in the intensive care stroke positioning study. ${ }^{23}$ Our results are in line with patients with subarachnoid haemorrhage in whom CBFV remained constant in the supine and $45^{\circ}$ patient body positions, regardless of the development of vasospasm. ${ }^{24}$

It is widely believed that a sudden $\mathrm{BP}$ reduction can affect $\mathrm{CBF}$ and render elderly participants or patients with cardiovascular disease vulnerable to orthostatic symptoms. ${ }^{25}$ The sitting position might be dangerous, especially with globally impaired cerebral autoregulation, because of the susceptibility of the penumbral regions to local hypoperfusion in the acute and subacute phases. However, our physiological results indicate that sitting is probably well tolerated in mild to moderately affected patients with stroke. In 2008, the 'A Very Early Rehabilitation trail' showed that very early out of bed mobilisation was safe and feasible in patients with stroke. ${ }^{26}$ This may be due to the counterbalancing effects of BP elevation, ICP reduction and increased venous return to stabilise cerebral perfusion pressure in the subacute phase. There is evidence for increased sympathetic activation in patients with acute stroke. ${ }^{27-29}$ Replication of this study should be considered in studying the (hyper)acute phase, longer sitting periods and using more sophisticated and direct methods that measure $\mathrm{CBF}$ regionally and estimate cerebral oxygen metabolism. ${ }^{30}$ Inclusion of more severely disabled patients (NIHSS around 10) would be preferable to being able to compare upright cerebral haemodynamics with the results from the ongoing 'early mobilisation' trails. ${ }^{26}$ Further research should also include more detailed (follow-up) information from extracranial and intracranial vessels pathology (using validated radiological or ultrasound criteria), recanalisation (proximal and distal), penumbra configuration and/or the presence of collaterals. ${ }^{31-34}$ Follow-up studies several days after stroke may be warranted because local and systemic compensatory mechanisms may become exhausted, but around that time influence on penumbral salvage may be limited. ${ }^{35}$ Our results do not hold for sitting to the standing mobilisation, which may provoke different (probably larger) haemodynamic responses. ${ }^{36}$

In the paucity of results for the early out of bed mobilisation stroke trails, ${ }^{37}$ we suggest continuation of early upright positioning in patients with stroke in the stroke unit in the subacute phase and propose that continuous CBF measurements during early activity out of bed (standing) could be helpful in assuring safe and early mobilisation in the stroke unit. ${ }^{36}$

\section{CONCLUSION}

In conclusion, upright positioning in the stroke unit of mildly to moderately affected patients with stroke does not give significant reduction of $\mathrm{CBF}$ and (frontal)cerebral oxygenation surrogates during the subacute phase on the stroke unit. Supine or Trendelenburg positioning seems not to augment $\mathrm{MCA} \mathrm{CBFV}_{\text {mean }}$ or improve neurological status.

Contributors MJA, JWE and PV contributed to design and conceptualisation of the study, interpretation of the data, and drafting and revision of the manuscript. RS and JDK contributed to the interpretation of the data and revision of the manuscript. BK was responsible for the revision of the manuscript.

Funding MJA is supported by the Netherlands Organisation for Health Research and Development.

\section{Competing interests None.}

Patient consent Obtained.

Ethics approval Medical Ethical Committee, University Medical Center Groningen, Groningen, the Netherlands.

Provenance and peer review Not commissioned; externally peer reviewed.

Data sharing statement The authors are willing to share the data, as well as the additional unpublished data of follow-up studies (day 3 after stroke) and dynamic autoregulation results. 
Open Access This is an Open Access article distributed in accordance with the Creative Commons Attribution Non Commercial (CC BY-NC 3.0) license, which permits others to distribute, remix, adapt, build upon this work noncommercially, and license their derivative works on different terms, provided the original work is properly cited and the use is non-commercial. See: http:// creativecommons.org/licenses/by-nc/3.0/

\section{REFERENCES}

1. Markus HS. Cerebral perfusion and stroke. J Neurol Neurosurg Psychiatry 2004;75:353-61.

2. Diserens $\mathrm{K}$, Michel P, Bogousslavsky J. Early mobilisation after stroke: review of the literature. Cerebrovasc Dis 2006:22:183-90.

3. Bernhardt J, Indredavik B, Dewey H, et al. Mobilisation 'in bed' is not mobilisation. Cerebrovasc Dis 2007;24:157-8.

4. Hill K. Australian Clinical Guidelines for Acute Stroke Management 2007. Int J Stroke 2008;3:120-9.

5. Tyson SF, Nightingale P. The effects of position on oxygen saturation in acute stroke: a systematic review. Clin Rehabil 2004:18:863-71.

6. McArthur KS, Quinn TJ, Higgins P, et al. Post-acute care and secondary prevention after ischaemic stroke. BMJ 2011;342:d2083.

7. Cumming TB, Thrift AG, Collier JM, et al. Very early mobilization after stroke fast-tracks return to walking: further results from the phase II AVERT randomized controlled trial. Stroke 2011;42:153-8.

8. Skarin M, Bernhardt J, Sjoholm A, et al. 'Better wear out sheets than shoes': a survey of 202 stroke professionals' early mobilisation practices and concerns. Int J Stroke 2011;6:10-15.

9. Wojner-Alexander AW, Garami Z, Chernyshev OY, et al. Heads down: flat positioning improves blood flow velocity in acute ischemic stroke. Neurology 2005;64:1354-7.

10. Bamford J, Sandercock P, Jones $L$, et al. The natural history of lacunar infarction: the Oxfordshire Community Stroke Project. Stroke 1987; 18:545-51.

11. Kolominsky-Rabas PL, Weber M, Gefeller O, et al. Epidemiology of ischemic stroke subtypes according to TOAST criteria: incidence, recurrence, and long-term survival in ischemic stroke subtypes: a population-based study. Stroke 2001;32:2735-40.

12. Poli L, Bo M, Secreto $\mathrm{P}$, et al. Age-related transcranial Doppler findings in the evaluation of cerebral circulation during postprandial and postural tests. Cerebrovasc Dis 1999;9:98-101.

13. Littell RC, Milliken GA, Stroup WW, et al. SAS system for mixed models. North Carolina: SAS Institute Inc, 1996.

14. Immink RV, van Montfrans GA, Stam J, et al. Dynamic cerebral autoregulation in acute lacunar and middle cerebral artery territory ischemic stroke. Stroke 2005;36:2595-600.

15. Giller CA, Bowman G, Dyer H, et al. Cerebral arterial diameters during changes in blood pressure and carbon dioxide during craniotomy. Neurosurgery 1993;32:737-41.

16. Bucher HU, Edwards $\mathrm{AD}$, Lipp $\mathrm{AE}$, et al. Comparison between near infrared spectroscopy and 133Xenon clearance for estimation of cerebral blood flow in critically ill preterm infants. Pediatr Res 1993;33:56-60.

17. Rasmussen $\mathrm{P}$, Dawson EA, Nybo L, et al. Capillary-oxygenation-leveldependent near-infrared spectrometry in frontal lobe of humans. J Cereb Blood Flow Metab 2007;27:1082-93.
18. Newell DW, Aaslid R, Lam A, et al. Comparison of flow and velocity during dynamic autoregulation testing in humans. Stroke 1994;25:793-7.

19. Hunter AJ, Snodgrass SJ, Quain D, et al. HOBOE (Head-of-Bed Optimization of Elevation) Study: association of higher angle with reduced cerebral blood flow velocity in acute ischemic stroke. Phys Ther 2011;91:1503-12.

20. Schwarz S, Georgiadis D, Aschoff A, et al. Effects of body position on intracranial pressure and cerebral perfusion in patients with large hemispheric stroke. Stroke 2002;33:497-501.

21. Jack Cl, Lee $\mathrm{T}$, Moyle $\mathrm{P}$, et al. The importance of posture in the early stages of stroke: its influence on cerebral perfusion. Age Ageing 2001;30:428.

22. Hargroves $\mathrm{D}$, Tallis $\mathrm{R}$, Pomeroy $\mathrm{V}$, et al. The influence of positioning upon cerebral oxygenation after acute stroke: a pilot study. Age Ageing 2008;37:581-5.

23. Dagal A, Lam AM. Cerebral autoregulation and anesthesia. Curr Opin Anaesthesiol 2009;22:547-52.

24. Zhang Y, Rabinstein AA. Lower head of the bed position does not change blood flow velocity in subarachnoid hemorrhage. Neurocrit Care 2011;14:73-6.

25. Lipsitz LA. Orthostatic hypotension in the elderly. N Engl J Med 1989;321:952-7.

26. Bernhardt J, Dewey $\mathrm{H}$, Thrift $\mathrm{A}$, et al. A very early rehabilitation trial for stroke (AVERT): phase II safety and feasibility. Stroke 2008;39:390-6.

27. Qureshi Al. Acute hypertensive response in patients with stroke: pathophysiology and management. Circulation 2008;118:176-87.

28. Korpelainen JT, Sotaniemi KA, Suominen K, et al. Cardiovascular autonomic reflexes in brain infarction. Stroke 1994;25:787-92.

29. Chamorro A, Amaro S, Vargas M, et al. Catecholamines, infection, and death in acute ischemic stroke. $J$ Neurol Sci 2007:252:29-35.

30. Ouchi Y, Yoshikawa E, Kanno T, et al. Orthostatic posture affects brain hemodynamics and metabolism in cerebrovascular disease patients with and without coronary artery disease: a positron emission tomography study. Neuroimage 2005;24:70-81.

31. Tsivgoulis G, Ribo M, Rubiera M, et al. Real-time validation of transcranial Doppler criteria in assessing recanalization during intra-arterial procedures for acute ischemic stroke: an international, multicenter study. Stroke 2013;44:394-400.

32. Bang OY, Saver JL, Buck BH, et al. Impact of collateral flow on tissue fate in acute ischaemic stroke. J Neurol Neurosurg Psychiatry 2008:79:625-9.

33. Wheeler HM, Mlynash M, Inoue M, et al. Early diffusion-weighted imaging and perfusion-weighted imaging lesion volumes forecast final infarct size in DEFUSE 2. Stroke 2013;44:681-5.

34. Tomsick T. TIMI, TIBI, TICI: I came, I saw, I got confused. AJNR Am J Neuroradiol 2007;28:382-4.

35. Reinhard M, Wihler C, Roth M, et al. Cerebral autoregulation dynamics in acute ischemic stroke after rtPA thrombolysis. Cerebrovasc Dis 2008;26:147-55.

36. Kim YS, Bogert LW, Immink RV, et al. Effects of aging on the cerebrovascular orthostatic response. Neurobiol Aging 2011;32:344-53.

37. Bernhardt J, Dewey H, Collier J, et al. A Very Early Rehabilitation Trial (AVERT). Int J Stroke 2006;1:169-71. 